\title{
Twin-twin transfusion syndrome: a five year review
}

\author{
Y C Seng, V S Rajadurai
}

\begin{abstract}
Objective-To determine the incidence, complications, management, and outcome in infants with twin-twin transfusion syndrome (TTTS) over a period of five years.

Methods-TTTS was diagnosed in monochorionic twins if one was pale and the other plethoric with a haemoglobin difference $\geqslant 5 \mathrm{~g} / 100 \mathrm{ml}$ and/or birthweight differences $\geqslant 15 \%$.

Results-Eighteen (6.2\%) of the 292 twin pairs had TTTS. Eight pairs $(44 \%)$ had the acute type and the rest $(56 \%)$ had the chronic type of TTTS. The mean (SEM) intrapair haemoglobin difference in the acute type was $4.8(2.1) \mathrm{g} / 100 \mathrm{ml}$ which gave a discordance of $7.1(4.6) \%$, whereas that in the chronic type was $6.9(2.9) \mathrm{g} / 100 \mathrm{ml}$ and $24.4(6.1) \%$ respectively. Infants with the acute type had a significantly higher incidence of vaginal delivery $(p<0.03)$, hypotension $(p<0.025)$, and respiratory distress $(p<0.01)$ compared with those with the chronic type. There was no significant difference in the incidence of anaemia, polycythaemia, asphyxia, hypoglycaemia, and hyperbilirubinaemia. Two recipients died in utero as the result of chronic TTTS, while their survivors developed spastic cerebral palsy. There were no neonatal deaths. Conclusions-TTTS, although uncommon, may have an adverse neurodevelopmental outcome especially if one twin dies in utero. Prompt recognition and management of the haemodynamic and haematological problems of infants with the acute types of TTTS will result in optimal neurodevelopmental outcome.

(Arch Dis Child Fetal Neonatal Ed 2000;83:F168-F170)
\end{abstract}

Department of Pediatrics, Kandang Kerbau Women's and Children's Hospital, Singapore

Y C Seng

Department of Neonatology

V S Rajadurai

Correspondence to: Dr Seng Yi-Chern Department of Pediatrics, Kandang Kerbau Women's and Children's Hospital, 100 Bukit Timah Road,

Singapore 229899

tgong@singnet.com.sg

Accepted 6 January 2000 direction, it was hypothesised that the third circulation predominates and results in the twin-twin transfusion syndrome (TTTS). When the cotyledon is supplied by an artery from one twin and drained by a vein from the other twin, there is potential for transfusion from the donor twin (on the arterial side) to the recipient twin (on the venous side). Consequently, the donor twin becomes anaemic, hypovolaemic, hypotensive, and hypoproteinaemic, undergoes intrauterine growth retardation, and occasionally develops oligohydramnios. In contrast, the recipient twin is heavier, polycythaemic, hypervolaemic, and faces complications of hyperviscosity of the blood such as cardiac failure, hyperbilirubinaemia, and intravascular thrombosis; polyuria may result in polyhydramnios. $^{3}$ The degree of severity of the twin transfusion is dependent on the duration of pregnancy, structural alterations of the vasculature (vessel calibre, quality of the anastomoses, and the presence/absence of vascular communication in the opposite direction to compensate for the haemodynamic imbalance), and chronicity of transfusion. ${ }^{8}$

The objective of this study was to determine the incidence and complications and to review our experience in the management and outcome of TTTS in a neonatal unit over a period of five years.

\section{Methods}

This is a retrospective review of all the twin pairs born in a neonatal unit in Kandang Kerbau Hospital during the period July 1990 to July 1995 . The data were collected by a manual search through 292 neonatal records during the study period.

Maternal data, antenatal ultrasound findings, obstetric and intrapartum complications, and placental morphology were assessed. Neonatal data included the mode of delivery, birth weight, gestation, Apgar scores, and details of resuscitation.

Clinical features of TTTS were studied, namely haemoglobin discrepancies, birthweight differences, presence of pallor, plethora, anaemia, polycythaemia, and hypotension. The diagnosis of TTTS was considered in monochorionic twins if any two of the following criteria were satisfied ${ }^{4}$ :

- intertwin haemoglobin difference $>5 \mathrm{~g} / 100$ $\mathrm{ml}$

- pallor in one twin and plethora in the other

- intertwin birthweight difference $\geqslant 15 \%$

The TTTS was considered to be acute if the birthweight discrepancy was less than $15 \%$ and 
if haemodynamic problems such as tachycardia or hypotension were present. The various types of treatment used were also analysed, namely plasma or normal saline infusion, inotropic support, whole blood or packed cell transfusion or partial plasma exchange transfusion for polycythaemia. Other significant neonatal problems were also reviewed, including perinatal asphyxia, hyaline membrane disease, transient tachypnoea of the newborn, congenital pneumonia, retinopathy of prematurity, necrotising enterocolitis, patent ductus arteriosus, intraventricular haemorrhage, and congenital malformations. After discharge from hospital, the infants were followed up in the outpatient clinic where the growth, development, and neurological sequelae were assessed.

\section{Results}

During the study period from June 1990 to June 1995, 292 twin pairs were admitted to our neonatology unit. In this study cohort, there were 130 female twin pairs, 149 male twin pairs, and 13 twin pairs of different sexes. Of these, 18 cases satisfied the diagnostic criteria for TTTS, giving an incidence of $6.2 \%$. Half of the 18 twin pairs were boys, and the rest were girls. Their mean (SEM) birth weight was 1865 (502) g (range 870-3020) and gestation was 33.6 (3.2) weeks (range 27-38). The index twin pairs were classified into acute and chronic types of TTTS; $44 \%$ had the acute type. The mean (SEM) intrapair haemoglobin difference in the acute type was $4.8(2.1) \mathrm{g} / 100$ $\mathrm{ml}$ which gave a discordance of 7.1 (4.6)\%, whereas that in the chronic type was 6.9 (2.9) $\mathrm{g} / 100 \mathrm{ml}$ and $24.4(6.1) \%$ respectively. Of the donor twins, 10 had anaemia and eight had hypotension requiring colloid or blood transfusion. Furthermore, seven donor twins had respiratory distress and three had perinatal asphyxia. Of the recipient twins, seven had polycythaemia with packed cell volume greater than $65 \%$, and three had respiratory distress. Moreover, two recipient twins had perinatal asphyxia, and one had hypotension. Three of the infants with polycythaemia needed partial plasma exchange transfusion. Of the recipient twins, two died in utero as the result of chronic TTTS.

Infants with the acute type of TTTS had a significantly higher incidence of vaginal delivery $(\mathrm{p}<0.03)$, hypotension $(\mathrm{p}<0.025)$, and respiratory distress $(\mathrm{p}<0.01)$ compared with those with the chronic type (table 1). There

Table 1 Comparison of neonatal problems in infants with acute and chronic types of twin-twin transfusion syndrome

\begin{tabular}{lccccc}
\hline Neonatal problems & $\begin{array}{l}\text { Acute } \\
(n=20)\end{array}$ & \multicolumn{4}{c}{$\begin{array}{l}\text { Chronic } \\
(n=16)\end{array}$} \\
\hline Mean gestation (weeks) & 33.1 & & 33.1 & \\
Caesarean section & 3 & $(15)$ & 8 & $(50)$ & \\
Vaginal delivery & 17 & $(85)$ & 8 & $(50)$ & $\mathrm{p}<0.03$ \\
Anaemia & 4 & $(20)$ & 5 & $(31)$ & \\
Polycythaemia & 5 & $(25)$ & 2 & $(13)$ & \\
Perinatal asphyxia & 5 & $(25)$ & 3 & $(19)$ & \\
Hypotension & 8 & $(40)$ & 1 & $(6)$ & $\mathrm{p}<0.025$ \\
Respiratory distress & 9 & $(45)$ & 1 & $(6)$ & $\mathrm{p}<0.01$ \\
Hypoglycaemia & 4 & $(20)$ & 4 & $(25)$ & \\
Hyperbilirubinaemia & 15 & $(75)$ & 7 & $(44)$ & \\
\hline
\end{tabular}

Values in parentheses are percentages.
Table 2 Follow up of infants with acute and chronic types of twin-twin transfusion syndrome and outcomes

\begin{tabular}{lc}
\hline Acute & $\mathrm{n}=20$ \\
Mortality & 0 \\
Developmental delay & 0 \\
Chronic & $\mathrm{n}=16$ \\
Survivors & $14(87.5)$ \\
Mortality (IUD) & $2(12.5)$ \\
Normal development & $10(72)$ \\
Delayed speech & $2(14)$ \\
Cerebral palsy with mental retardation & $2(14)$ \\
\hline
\end{tabular}

Values in parentheses are percentages.

IUD, intrauterine death.

was no significant difference in the incidence of anaemia, polycythaemia, asphyxia, hypoglycaemia, and hyperbilirubinaemia.

All the survivors were followed up for a period of two years. There were no neonatal deaths among the study cohort. Physical growth was assessed from serial measurements of height, weight, and occipital frontal circumference. Neurodevelopment was also monitored from routine assessment of motor (fine/gross), visual, speech, and auditory skills. The physical and neurological development of each survivor was monitored at the ages of 3 months, 6 months, 9 months, 12 months, 18 months, and 2 years. Three pairs of affected twins were lost to follow up (one pair of which requested to be followed up by their private practitioner after six months and two pairs defaulted follow up after one year).

Overall, two of the 34 survivors with TTTS suffered neurological sequelae. All 16 infants who had the acute type showed normal growth and development on follow up (table 2). Of the 18 survivors who had the chronic type, two developed spastic cerebral palsy (one had spastic quadriplegia and the other had spastic diplegia). These two twins were donor twins whose other twin had died in utero.

\section{Discussion}

TTTS is a serious complication of monozygotic monochorionic twins resulting from a transplacental vascular communication. ${ }^{1}$ The incidence of TTTS in our study was $6.2 \%$, whereas the reported incidence varies between 5 and $15 \%$. The extreme variant of TTTS is the "stuck twin" phenomenon where one fetus lies against the uterine wall in a severely oligohydramniotic sac while the co-twin lies in a severely polyhydramniotic sac. This complication has been reported in $8 \%$ of twin pregnancies. $^{5}$ The most serious problem with vascular shunting is the formation of an acardiac or amorphous fetus when large artery-artery and vein-vein anastomoses form between the anomalous fetus and its usually normal co-twin. Blood enters the recipient through the umbilical artery and leaves the umbilical vein to drain into a large placental vein-vein anastomosis. Low perfusion pressure and desaturation result in an acardiac fetus. The normal twin, while perfusing the circulation of both twins, may develop cardiac hypertrophy, congestive cardiac failure, or hydrops. ${ }^{6}$

Our study has shown that when TTTS results in the intrauterine death of one of the fetuses, the surviving twin has an adverse neu- 
rodevelopmental outcome. In the two cases in which the monochorionic twins had died in utero, cerebral palsy with mental retardation was the eventual outcome. This is consistent with current literature, which attributes the increased risk of morbidity and mortality in the survivor to intrauterine disseminated coagulation initiated by the passage of thromboplastic material from the dead twin through vascular anastomoses. Furthermore, recent data have suggested that rapid and profound haemodynamic alterations at the time of the death of one twin could be responsible for infarction, necrosis, cystic periventricular leucomalacia, and renal damage in the survivors. ${ }^{7}$ In the series of 14 cases studied by Cincotta et al ${ }^{9}$, periventricular leucomalacia, oliguria, and renal failure were thought to be secondary to hypotension caused by acute transfusion from the donor to the recipient.

In our study, the infants who had TTTS have been further subclassified into acute and chronic types. When the birthweight discrepancy between twins is small, the transfusion is considered to have occurred acutely. ${ }^{6} \mathrm{~A}$ distinction between the acute and chronic forms of the syndrome can be made on the basis of weight discrepancy and haemodynamic changes. Infants with the chronic form have discrepancies in birth weight exceeding $15 \%$ and the peripheral blood film of the donor twin may show hypochromic microcytic anaemia and erythroblastosis. The acute forms may be one of the emergencies encountered in the delivery room. Severe anaemia and shock may occur in the donor and require urgent transfusion of blood. The recipient twin may be in cardiac failure and require urgent reduction of his/her blood volume by withdrawal of blood from the umbilical vein.

Cord haemoglobin may be misleading in the acute forms of the syndrome, as they may be normal with no intrapair discrepancy. In such instances, a full blood count performed between six and 12 hours after delivery will show the characteristic intrapair discrepancy. Acute severe TTTS occurs in $1 \%$ of monochorionic twin pregnancies and may present as acute hydramnios resulting in preterm labour or premature rupture of membranes. A high mortality ranging between 79 and $100 \%$ has been reported for twins with the acute types presenting at $18-26$ weeks. ${ }^{6}$ All eight twin pairs in our study who had the acute types survived. There were no cases of acute polyhydramnios in our cohort and the rapid and profound haemodynamic changes probably occurred in the intrapartum period. Interestingly, a significantly higher incidence of vaginal delivery was noted in cases of acute TTTS. Prompt recognition and management of hypotension, anaemia, and polycythaemia is essential to optimise neurodevelopmental outcome as shown in the above study.

In antenatally diagnosed cases, a variety of interventional strategies have been described. ${ }^{2}$ Repeated decompression amniocentesis has been attempted by several authors for the relief of maternal discomfort and also to improve fetal outcome. However, the overall perinatal mortality was $54.7 \%$ and it had little efficacy in the treatment of the affected fetuses. ${ }^{1}$ Another treatment is selective feticide, which has been described by several authors such as Wittmann et al, ${ }^{10}$ Weiner, ${ }^{11}$ and Chitkara et al. ${ }^{12}$ However, their attempt to ligate the umbilical cord was not successful. This treatment is not recommended because of the potentially poor neurodevelopmental outcome in the surviving twin.

Treating the mother with digoxin when the recipient twin is showing signs of cardiac failure has had favourable results. ${ }^{1}$ Nonsteroidal anti-inflammatory agents such as indomethacin have been tried but not shown to improve fetal outcome.

Placental surgery has been considered as an alternative mode of treatment in TTTS. Delia et $a l^{13}$ attempted fetoscopically directed occlusion of the placental vessels with a neodymiumYAG laser in four ewes. They later used the same method on three pairs of affected twins, two of which survived.

In conclusion, although the incidence of TTTS is low $(6.2 \%)$, it is an important phenomenon in view of its association with morbidity in the affected twins. It is also shown to result in unfavourable neurodevelopmental outcome in the surviving twin if its co-twin dies in utero. Furthermore, the chronic type of TTTS is also associated with more adverse outcome. Prompt recognition and management of the haemodynamic and haematological problems of infants with the acute types of TTTS will result in optimal neurodevelopmental outcome.

1 Blickstein I. The twin-twin transfusion. Obstet Gynecol 1990;76:714-22.

2 Urig MA, Clewell WH, Elliott JP. Twin-twin transfusion. Am $\mathcal{F}$ Obstet Gynecol 1990;163:1522-6.

3 Urig MA, Clewell WH, Elliott JP. Aggressive therapeutic amniocentesis for treatment of twin-twin transfusion. Obstet Gynecol 1991;77:537-40.

4 Shah DM, Chaffin D. Perinatal outcome in very preterm births with twin-twin transfusion. Am f Obstet Gynecol 1989;161:1111-13.

5 Rehan VK, Menticoglou SM. Mechanism of visceral damage in fetofetal transfusion syndrome. Arch Dis Child 1995;73:F48-50.

6 McCulloch K. Neonatal problems in twins. Clin Perinatol 1988;15:141-54.

7 Fusi L, McParland P, Fisk N, Wigglesworth J. Acute twintwin transfusion: a possible mechanism for brain-damaged survivors after intrauterine death of a monochorionic twin. Obstet Gynecol 1991;78:517-20.

8 Bardawil WA, Reddy RL, Bardawil LW. Placental considerations in multiple pregnancy. Clin Perinatol 1988;15:13-39.

9 Cincotta R, Oldham J, Sampson A. Antepartum and postpartum complications of twin-twin transfusion. Aust NZ Z Obstet Gynaecol 1996;36:303-8.

10 Wittmann BK, Farquharson DF, Thomas WDS, Baldwin VJ, Wadsworth LD. The role of feticide in the management of severe twin transfusion syndrome. Am $\mathcal{F}$ Obstet Gynecol 1986;155:1023-6.

11 Weiner CP. Diagnosis and treatment of twin to twin transfusion in the mid-second trimester of pregnancy. Fetal Therapy 1987;2:71-4.

12 Chitkara U, Berkowitz RL, Wilkins IA, Lynch L, Mehalek $\mathrm{KE}$, Alveraz M. Selective second-trimester termination of the anomalous fetus in twin pregnancies. Obstet Gynecol 1989;73:690-4

13 DeLia JE, Rogers JG, Dixon JA. Treatment of placental vasculature with neodymium-yttrium-aluminium-garnet laser via fetoscopy. Am $\mathcal{F}$ Obstet Gynecol 1985;151:1126-7. 